\title{
In-Plane Molecular Rotational Dynamics at a Negatively Charged Surfactant/Aqueous Interface
}

\author{
Xiaoming Shang, Kim Nguyen, Yi Rao, and Kenneth B. Eisenthal* \\ Department of Chemistry, 3000 Broadway, Columbia University, New York, New York 10027
}

Received: August 14, 2008; Revised Manuscript Received: September 23, 2008

\begin{abstract}
The in-plane rotational dynamics of the molecular probe coumarin 314 (C314) at the negatively charged surfactant sodium dodecyl sulfate (SDS)/aqueous interface was measured using femtosecond time-resolved second-harmonic spectroscopy. The in-plane orientational time constant at an SDS surface coverage of 100 $\AA^{2}$ per SDS molecule is $348 \pm 12 \mathrm{ps}$, which is comparable to the out-of-plane reorientational time of $383 \pm$ 9 ps at the same SDS density. At $100 \AA^{2}$, the SDS surfactant forms a homogeneous monolayer because it is in the liquid-condensed region of the surface pressure phase diagram. The rotation dynamics is slower than at the neat air/water interface, where the in-plane orientational time constant is $304 \pm 8$ ps and the out-ofplane reorientational time constant is $336 \pm 6$ ps. For purposes of comparison, we found that the orientational relaxation time of C314 in bulk water is $262 \pm 10$ ps. There is clear evidence for strong C314-SDS interfacial interactions based on the significant dependence of equilibrium properties on the SDS density. For example, large changes are seen in the C314 interfacial molecular orientation, marked changes in the SHG surface spectra, and changes in the surfactant phase diagram. Unlike these equilibrium properties, neither the inplane nor the out-of-plane rotations are strongly affected by interfacial SDS. The relative insensitivity of interfacial molecular rotations to the presence of anionic surfactants has implications to molecular rotations at biological cell/membrane aqueous interfaces, most of which contain anionic phospholipids.
\end{abstract}

\section{Introduction}

The asymmetric environment at an interface presents a unique chemical microcosm for molecular adsorbates. Due to both the change of solvent properties at the interface and the inherent anisotropy of the environment, molecular interactions and motions at the interface are anticipated to differ from that of the bulk liquid. ${ }^{1-6}$ Unlike the isotropic rotational motions of solutes in bulk water, the asymmetric surface potential at the interface restricts the rotational motions of adsorbed solutes. Interfacial molecules exhibit structure-based directionality at an aqueous/air interface, with one end of the molecule preferentially immersed in the condensed water layer and the other end chiefly found exposed to the vapor phase. This directionality is expected to be reflected in different interfacial molecular rotational motions that are in the plane of the interface versus motions out of the interfacial plane..$^{7-13}$ For the investigation of species with a measurable solubility in the bulk, the overwhelming bulk contribution often precludes the use of linear spectroscopic approaches to measure surface properties. The surface specificity afforded by second harmonic generation (SHG) provides a robust optical means to study the dynamics of molecules present at interfacial regions. ${ }^{14-16}$ SHG has been used extensively to probe interfacial phenomena at gas-liquid,,$^{8,17-19}$ gas - solid, ${ }^{20,21}$ liquid-solid, ${ }^{22-26}$ and liquid-liquid ${ }^{27-31}$ interfaces in real time, in situ, and with molecular specificity.

There are arguably no interfaces of greater importance than the air/aqueous interface and the aqueous/membrane interface of a living cell. One can argue that the inner and outer aqueous membrane interfaces of biological cells are the most complex of all interfaces, as host and active participant in the myriad of complex molecular dynamical interactions which support life

\footnotetext{
*E-mail:kbe1@columbia.edu.
}

itself. The lipid bilayers that provide the basic structure of the mammalian cell consist of $4-30 \%$ anionic phospholipids. ${ }^{32}$ Amphiphilic lipid surfactant films formed atop liquids are commonly used as simple membrane-mimetic systems for interfacial study. ${ }^{3,5,6,11,33-35}$ The addition to $\mathrm{SDS}\left(\mathrm{CH}_{3}\left(\mathrm{CH}_{2}\right)_{11^{-}}\right.$ $\mathrm{OSO}_{3}{ }^{-} \mathrm{Na}^{+}$) to a saturated solution of coumarin 314 (C314) results in an anionic monolayer at the water interface, where the negatively charged sulfate groups are immersed in the water phase and the hydrophobic dodecane tails extend into the air. ${ }^{36}$ The sodium counterions remain in the bulk solution. The charge density at the interface upon introduction of an anionic monolayer changes the ordering of water molecules at the interface, ${ }^{3,37}$ which, in turn, are likely to affect the equilibrium and dynamical properties of $\mathrm{C} 314$ probe molecules present at the interface.

Previous studies in this laboratory have reported slower orientation times of rhodamine $6 \mathrm{G}$ and coumarin 314 (C314) at the air/water interface relative to their bulk values. ${ }^{7,38,39}$ The C314 out-of-plane relaxation time of 336 ps is significantly slower than its value of $262 \pm 10$ ps in bulk water. ${ }^{40}$ The outof-plane polar angle, $(\theta)$, relaxation time of $\mathrm{C} 314$ at the interface indicates that rotational friction at the air $/ \mathrm{H}_{2} \mathrm{O}$ interface is greater than in bulk water (Figure 1). Of direct relevance to the change in rotational dynamics are the orientational arrangements of water molecules and the hydrogen bonding structure of interfacial water. Second harmonic phase measurements have shown that the water molecules are preferentially aligned with the hydrogens directed toward the bulk water and the oxygen toward the vapor phase. ${ }^{41}$ On the basis of the weakness of the second harmonic signal from the bare air/aqueous interface, it was suggested that the water molecules prefer an alignment that is close to being flat, which would reduce the SHG signal. Sum frequency generation (SFG) measurements indicate that $20-30 \%$ 


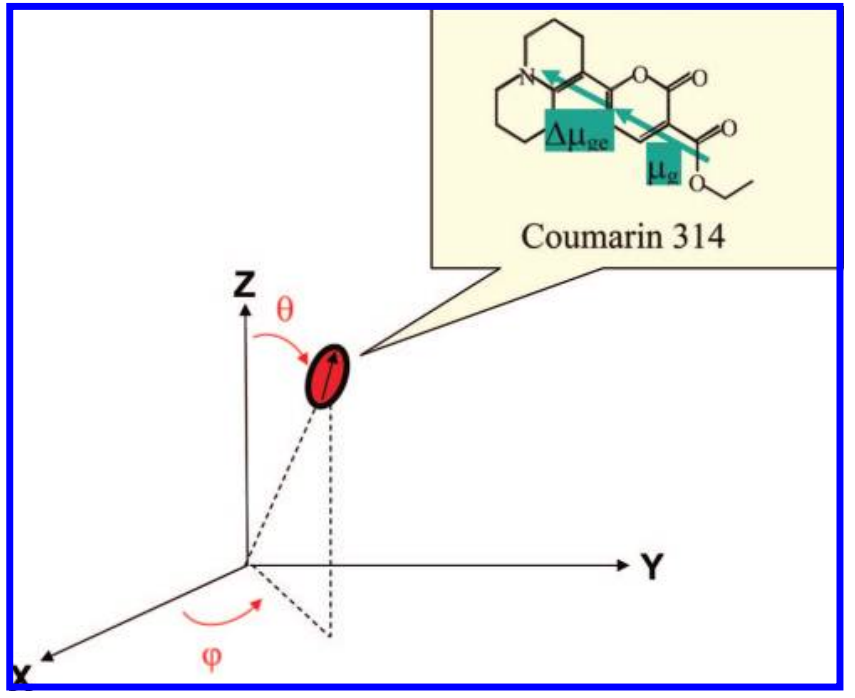

Figure 1. Out-of-plane $(\theta)$ and in-plane $(\varphi)$ rotation angles with respect to the laboratory coordinate system. The arrow on the C314 molecule shows the direction of the permanent dipole moment and the transition dipole moment of the $\mathrm{S}_{0}$-to- $\mathrm{S}_{1}$ transition. $\Delta \mu=\mu_{\mathrm{e}}-\mu_{\mathrm{g}}$, where $\mu_{\mathrm{e}}$ and $\mu_{\mathrm{g}}$ are the permanent dipole moments of the excited- and ground-state molecules.

of the water molecules have "free" hydrogens, that is, nonhydrogen bonded, that project into the vapor phase. ${ }^{1,37}$ Extensive SFG measurements of the interface water have shown that the structure of water is further altered at charged interfaces, 3,37 where the increased hydrogen bonding is thought to give rise to "icelike" interfacial water structures with increasing anionic surfactant surface density.

The underlying idea of the pump-probe method to measure orientational relaxation is to perturb the equilibrium orientational distribution of ground-state molecules and to monitor the return of the ground-state molecules that were not excited by the pump to their equilibrium orientational distribution and, similarly, for the newly created excited-state molecules to reorient toward their equilibrium orientational distribution. The perturbation of the initial equilibrium ground-state orientation is effected by the polarized pump light preferentially exciting molecules whose transition moments are oriented along the polarization direction of the pump light. In this paper, we report the in-plane rotation dynamics of C314 at a negatively charged surfactant/aqueous interface, which is of special interest, one reason being that most biological cell membranes are negatively charged. ${ }^{32}$ Two aqueous interfaces were probed: the surfactant free air/water interface and the $100 \AA^{2}$ per molecule/SDS water interface, where the monolayer is in a homogeneous liquid condensed region of the phase diagram (Figure 2). The reason that intermediate surfactant densities were not investigated in these studies is that at such densities, the surface is not homogeneous, being in the gas-liquid coexistence region of the phase diagram, which at the air/SDS water interface consists of islands of SDS molecules and gas phase "free" SDS molecules. Thus, C314 adsorbates experience different interfaces that range from the air/SDS - water islands to the "free" SDS molecules at the air/ water interface. In contrast, at the SDS density of $100 \AA^{2}$, the SDS monolayer is in the liquid condensed region of the diagram, which means that the $\mathrm{C} 314$ adsorbates experience a homogeneous air/SDS - water interface.

Isolation of the in-plane orientational component of $\mathrm{C} 314$ molecular interfacial rotation has previously been described ${ }^{7}$ the current analysis is a modification of that work and corrects

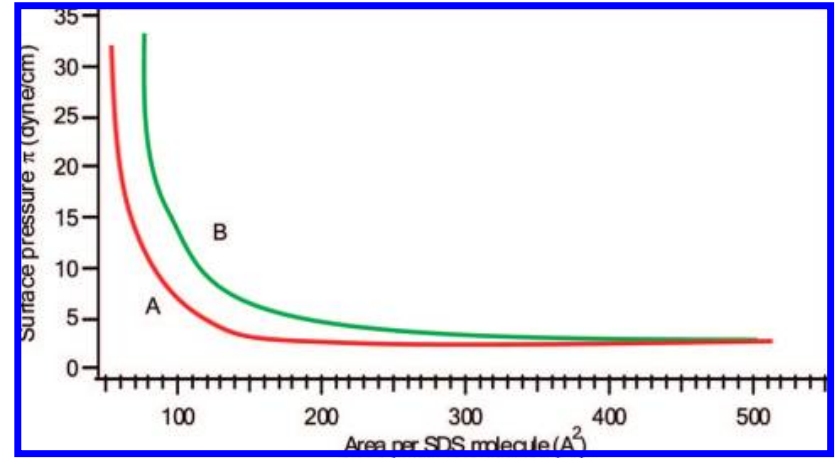

Figure 2. Surface pressure vs area per SDS molecule phase diagram ${ }^{6}$ (A) without coumarin 314 , and (B) with a $15 \mu \mathrm{M}$ coumarin 314 bulk water concentration.

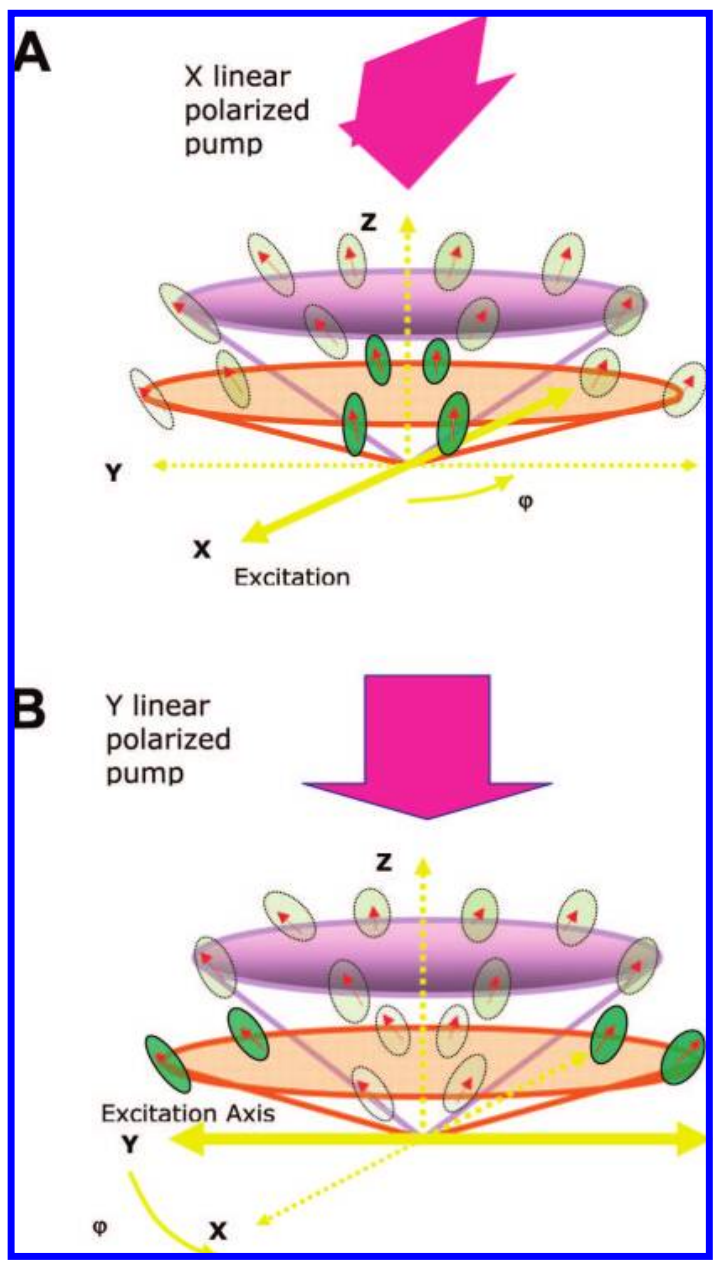

Figure 3. Schematic showing the effect of a normal incidence (A) $X$-polarized linear pump pulse and (B) $Y$-polarized linear pump pulse on the orientational distribution of the ground and excited state of $\mathrm{C} 314$ molecules at an air/aqueous interface. The darkened C314 molecules denote the excited-state molecules.

an error in the calculated value for in-plane rotation. In brief, a linearly polarized pump pulse is incident normal to the interfacial plane, along the $Z$-axis, which the out-of-plane angle $\theta$ subtends (Figure 3). The in-plane angle $\varphi$ lies in the $X-Y$ plane of the laboratory axis, which is the interfacial plane to which C314 molecules are adsorbed. C314 symmetry is approximated as uniaxial, and its in-plane angular $\varphi$ distribution at equilibrium is isotropic in the interfacial plane because the surface of a liquid is isotropic. The anisotropy of the interface occurs in the direction normal to the interface, where up and down clearly 
differ. Pump light that is linearly polarized along the $X$-axis ( $X$-pump) preferentially excites ground-state C314 molecules whose absorption is along the $X$-axis (Figure $3 \mathrm{~A}$ ), whereas the linearly polarized $Y$-pump does the same with the $Y$-axis C314 population (Figure 3B). Excitation with $X$ and $Y$ pump light breaks the isotropy of the in-plane orientational distribution. For experiments in which the pump light is polarized along the laboratory $X$ axis, there is a resultant excess of excited molecules orientated in the $X$ direction and a reduction of ground-state molecules oriented in the $X$ direction. In this discussion, orientation of the $\mathrm{C} 314$ molecule is given by the orientation of the $S_{0} \rightarrow S_{1}$ transition, which is also the orientation of the C314 dipole moment with respect to the surface normal. Following the pump excitation, the excited molecules will rotate away from the $X$ axis toward larger $\varphi$ and also toward smaller values of $\theta$. The ground-state molecules will rotate toward the ground-state molecule-depleted $X$-axis; that is, to smaller values of $\varphi$ as well as toward smaller values of $\theta$. The in- plane rotations following $Y$-pumping will be opposite to that of $X$-pumping. The out-ofplane orientational distribution is the same for $Y$ - and $X$ pumping. The probability expressions for the excitation of ground-state C314 molecules initially oriented at angles $\varphi_{0}$ and $\theta_{0}$ yield for $X$ and $Y$ linear polarized pumping expressions 1 and 2, respectively. For later use, we observe that if the pump light is circularly polarized, the in-plane isotropy of groundand excited-state molecules is maintained; that is, no $\varphi_{0}$ dependence is present in expression 3 .

$$
\begin{gathered}
\left|\vec{\mu} \cdot \vec{E}_{X}\right|^{2}=\mu^{2} E_{X}{ }^{2} \sin ^{2} \theta_{0} \cos ^{2} \phi_{0} \\
\left|\vec{\mu} \cdot \vec{E}_{Y}\right|^{2}=\mu^{2} E_{Y}^{2} \sin ^{2} \theta_{0} \sin ^{2} \phi_{0} \\
\left|\vec{\mu} \cdot \vec{E}_{C^{2}}\right|^{2}=\mu^{2} E_{C}{ }^{2} \sin ^{2} \theta_{0}
\end{gathered}
$$

$\mu$ is the molecular transition dipole moment, and $E$ is the electric field amplitude of the polarized pump pulse. The initial in-plane excitation distribution for the $\mathrm{X}$-axis is proportional to $\cos ^{2} \varphi_{0}$, whereas that for the $Y$-axis is proportional to $\sin ^{2}$ $\varphi_{0}$. Note that the out-of-plane component, which is proportional to $\sin ^{2} \theta_{0}$, is common to both the $X$ and $Y$ linear pump polarizations. In contrast we note that for the circularly polarized pumping the excitation distribution of C314 molecules depends only on the out-of-plane angle $\theta_{0}$. Following the orientational anisotropy induced by the pump light, the ground and excited molecules rotate toward their equilibrium orientations. To probe the in-plane rotational motions, the out-of-plane and in-plane orientational dynamics must be differentiated. When the pump light is circularly polarized, the in-plane isotropy is maintained, and therefore, the SHG dynamics depends only on $\theta$; that is, we are monitoring the out-of-plane rotations only. With respect to $X$ and $Y$ pump light, the rotational motions depend on both $\theta$ and $\varphi$; that is, the out-of-plane and in-plane rotations. We assume that the in-plane motions do not depend on $\theta$, which is plausible if the out-of-plane orientational distribution is not very large. This assumption is supported by fluorescence studies of the out-of-plane and in-plane motions of acridine orange at various aqueous/organic liquid interfaces. ${ }^{13}$ Because the structure of the liquid interface is isotropic, the out-of-plane rotation $(\theta)$ of the solute adsorbate is necessarily independent of the $\varphi$ orientation of the molecule. However this does not require that the in-plane rotation $\varphi$ be independent of the $\theta$ orientation of the molecule. In this paper, we will treat the $\theta$ and $\varphi$ motions as independent, bearing in mind that it is an assumption. We use the following expressions to describe the dynamics with $X$ and $Y$-pumping,

$$
\begin{aligned}
& E_{X}{ }^{2 \omega}=\left(a_{X}+b_{X} \mathrm{e}^{-t \tau_{\phi}}\right)\left(a_{X}{ }^{\prime}+b_{X}{ }^{\prime} \mathrm{e}^{-t \tau_{\theta}}\right) \\
& E_{Y}{ }^{2 \omega}=\left(a_{Y}+b_{Y} \mathrm{e}^{-t \tau_{\phi}}\right)\left(a_{Y}{ }^{\prime}+b_{Y}{ }^{\prime} \mathrm{e}^{-t \tau_{\theta}}\right)
\end{aligned}
$$

$E_{X}{ }^{2 \omega}$ and $E_{Y}{ }^{2 \omega}$ are the second harmonic fields generated by the probe pulse for $X$ and $Y$ pumping; $a_{x}, b_{x}, a_{x}{ }^{\prime}, b_{x}{ }^{\prime}, a_{y}, b_{y}, a_{y}{ }^{\prime}$, $b_{y}{ }^{\prime}$ are constants; and $\tau_{\theta}$ and $\tau_{\varphi}$ are the time constants for the out-of-plane and in-plane motions, respectively. The expressions for $E_{X}{ }^{2 \omega}$ and $E_{Y}{ }^{2 \omega}$ can be simplified by the following considerations. Because the out-of-plane $(\theta)$ excitation probabilities are the same for $X$ and $Y$ pumping (eqs 1 and 2), the terms describing the out-of-plane dynamics for both cases must be the same. Thus, $a_{X}{ }^{\prime}=a_{Y}{ }^{\prime}=a^{\prime}$ and $b_{x}{ }^{\prime}=b_{Y}{ }^{\prime}=b^{\prime}$,

$$
\begin{aligned}
& E_{X}{ }^{2 \omega}=\left(a_{X}+b_{X} \mathrm{e}^{-t \tau_{\phi}}\right)\left(a^{\prime}+b^{\prime} \mathrm{e}^{-t \tau_{\theta}}\right) \\
& E_{Y}{ }^{2 \omega}=\left(a_{Y}+b_{Y} \mathrm{e}^{-t \tau_{\phi}}\right)\left(a^{\prime}+b^{\prime} \mathrm{e}^{-t \tau_{\theta}}\right)
\end{aligned}
$$

Further simplification of eqs 6 and 7 can be made by imposing the requirement that at times long relative to the rotational relaxation times, the dynamics for $X$ - and $Y$-pumping should reach the same signal level. Therefore, $a_{x}$ equals $a_{y}$. Using these requirements, one can rewrite eqs 6 and 7 as

$$
\begin{aligned}
& E_{X}{ }^{2 \omega}=\left(a+b_{X} \mathrm{e}^{-t \tau_{\phi}}\right)\left(a^{\prime}+b^{\prime} \mathrm{e}^{-t \tau_{\theta}}\right) \\
& E_{Y}{ }^{2 \omega}=\left(a+b_{Y} \mathrm{e}^{-t \tau_{\phi}}\right)\left(a^{\prime}+b^{\prime} \mathrm{e}^{-t \tau_{\theta}}\right)
\end{aligned}
$$

Because the sum of the $X$ - and $Y$-pumped excitation probabilities (eqs 1 and 2 ) is equal to the circularly polarized excitation probability (eq 3), it follows that the observed rotational dynamics with circularly polarized pumping must be the sum of the rotational dynamics obtained with $X$ and $Y$ pumping. In this way, we obtain $b_{X}=-b_{Y}$. Equations 8 and 9 become

$$
\begin{aligned}
& E_{X}{ }^{2 \omega}=\left(a+b \mathrm{e}^{-t \tau_{\phi}}\right)\left(a^{\prime}+b^{\prime} \mathrm{e}^{-t \tau_{\theta}}\right) \\
& E_{Y}{ }^{2 \omega}=\left(a-b \mathrm{e}^{-t \tau_{\phi}}\right)\left(a^{\prime}+b^{\prime} \mathrm{e}^{-t \tau_{\theta}}\right)
\end{aligned}
$$

By examining eqs 10 and 11 , one may conclude that the ratio of $E_{X}{ }^{2 \omega}$ to $E_{Y}{ }^{2 \omega}$ contains the information related only to in-plane dynamics,

$$
\frac{E_{X}{ }^{2 \omega}}{E_{Y}{ }^{2 \omega}}=\frac{a+b \mathrm{e}^{-t \tau_{\phi}}}{a-b \mathrm{e}^{-t \tau_{\phi}}}=\frac{1+a / b \mathrm{e}^{-t \tau_{\phi}}}{1-a / b \mathrm{e}^{-t \tau_{\phi}}}
$$

In eq $12, a / b$ can be treated as one constant, denoted as $A$. Equation 12 becomes

$$
\frac{E_{X}^{2 \omega}}{E_{Y}^{2 \omega}}=\frac{1+A \mathrm{e}^{-t \tau_{\phi}}}{1-A \mathrm{e}^{-t \tau_{\phi}}}
$$

By fitting the dynamics to eq 13, we extract the in-plane rotation time, $\tau_{\varphi}$.

A brief description of the pump-probe experiment is given in the following discussion, starting with the $X$-polarized pump experiment. The orientational distributions of ground- and excited-state molecules, which are generated by a one-photon resonant polarized pump pulse, are not equilibrium orientational distributions. Therefore, following photoexcitation, the groundand excited-state molecules will undergo rotational diffusion toward their respective equilibrium orientations. The $\mathrm{SH}$ polarization, $P_{2 \omega}(t)$, induced by the probe pulse of frequency $\omega$ at a time $t$ after the pump pulse $(t=0)$ can be written as

$$
\mathrm{P}^{(2)}(t \geq 0)=\left(\chi_{\mathrm{g}}^{(2)}(t)+\chi_{\mathrm{e}}^{(2)}(t)\right) E(\omega) E(\omega)+\chi_{\mathrm{NR}}^{(2)} E(\omega) E(\omega)
$$

where $\chi_{\mathrm{g}}^{(2)}(t)$ is the second order susceptibility of the molecules in the ground state at time $t$, and $\chi_{\mathrm{e}}^{(2)}(t)$ is the corresponding 
susceptibility of excited-state molecules, and $\chi_{\mathrm{NR}}^{(2)}$ is the nonresonant contribution from all molecules at the interface. This latter term is negligible compared with the first term and will be omitted. The susceptibility can be related to the second-order hyperpolarizabilities, $\alpha^{(2)}$, of the ground- and excited-state molecules and their respective populations, $N_{\mathrm{g}}$ and $N_{\mathrm{e}}$. Thus,

$$
\mathrm{P}^{(2)}(t \geq 0)=\left\langle N_{g}(\theta, \phi ; t) \alpha_{\mathrm{g}}^{(2)}+N_{\mathrm{e}}(\theta, \phi ; t) \alpha_{\mathrm{e}}^{(2)}\right\rangle E(\omega) E(\omega)
$$

where $N_{\mathrm{g}}(\theta, \phi, t)$ and $N_{\mathrm{e}}(\theta, \phi, t)$ are the number of groundstate molecules at $(\theta, \varphi)$ at time $t$ within the angular volume element $\mathrm{d} \Omega$. The terms inside the bracket include the probability of a polarized pump pulse exciting a ground-state molecule oriented at $\left(\theta_{0}, \varphi_{0}\right)$ at time $t=0$, and the evolution of the initial ground- and excited-state molecules rotating from $\left(\theta_{0}, \varphi_{0}\right)$ at $t$ $=0$ to $(\theta, \varphi)$ at $t$. Because the rotational dynamics is more than an order of magnitude faster than the excited-state lifetime, we neglect the decay of excited-state molecules. The quantity inside the bracket is then ensemble-averaged over the angles $(\theta, \varphi)$ to give $\mathrm{P}^{(2)}(t)$, which generates the second harmonic field, $E_{2 \omega}(t)$. The differences between $X, Y$, and circularly polarized pump light appearing in eq 15 are due solely to the different excitation probabilities given in eqs $1-3$. As an example, let us consider the case in which the hyperpolarizabilities, $\alpha^{(2)}$, are uniaxial along a given molecular axis, which turns out is the case for $\mathrm{C} 314$ at the wavelength of the probe light used in our experiments. This was demonstrated by our showing that the C314 orientational dynamics is the same for the $\chi_{x z x}^{(2)}$ and $\chi_{z x x}^{(2)}$ susceptibility elements, from which it follows that the symmetry of the C314 hyperpolarizability is uniaxial. ${ }^{7}$ Measurement of the $X$ component of the second harmonic light arising from the $\chi_{x z x, \mathrm{~g}}^{(2)}(t)$ ground- and $\chi_{x z x, \mathrm{e}}^{(2)}(t)$ excited-state molecules, generated by probe light incident at an angle of $70^{\circ}$ with respect to the surface normal, and having polarization components along the $X$ and $Z$ axes, results in the following expression for the excitedstate contribution to $\mathrm{P}_{X}^{(2)}(t)$.

$$
\begin{gathered}
\mathrm{P}_{X}^{(2)}(t)_{\mathrm{e}}=E_{X}(\omega) E_{Z}(\omega) \int_{\theta} \int_{\phi} \int_{\theta_{0}} \int_{\phi_{0}} N w_{\mathrm{g}}\left(\theta_{0}, \phi_{0} ; t<0\right) \times \\
k\left|\vec{\mu} \cdot E_{\text {pump }}\right|^{2} G_{\mathrm{e}}\left(\theta_{0}, \phi_{0} ; 0 \theta, \phi ; \mathrm{t}\right) \times \\
\alpha_{\xi \xi \xi, e}^{(2)} \sin ^{2} \theta \cos \theta \cos ^{2} \phi \sin \theta_{0} \mathrm{~d} \theta_{0} \mathrm{~d} \phi_{0} \sin \theta \mathrm{d} \theta \mathrm{d} \phi
\end{gathered}
$$

$\mathrm{P}_{X}^{(2)}(t)_{\mathrm{e}}$ is the excited-state contribution to the total polarization $\mathrm{P}_{X}^{(2)}(t) ; N$ is the total number of molecules at the interface; $w_{\mathrm{g}}\left(\theta_{0}\right.$, $\left.\varphi_{0} ; t<0\right)$ is the probability per angular volume that a groundstate molecule has the orientation $\theta_{0}, \varphi_{0}$ at a time before the pump pulse, $(t<0) ; k\left|\vec{\mu} \cdot \vec{E}_{\text {pump }}\right|^{2}$ is the probability of excitation of a ground-state molecule whose transition moment vector is at angles $\theta_{0}, \varphi_{0}$ with the electric field of the pump pulse $\vec{E}_{\text {pump }}$; $k$ is a constant; $\alpha_{\xi \xi \xi,}^{(2)}$ is the uniaxial hyperpolarizability of the excited molecule along the axis $\xi$; and $G_{\mathrm{e}}\left(\theta_{0}, \phi_{0} ; 0 \mid \theta, \varphi ; t\right)$ is the rotation function that brings the excited molecule from $\theta_{0}$, $\varphi_{0}$ at $t=0$ to $\theta, \varphi$ at time $t$. There is an analogous expression for the ground-state contribution to $\mathrm{P}_{X}^{(2)}(t)$. The ground-state hyperpolarizability $\alpha_{\xi \xi \xi, \mathrm{g}}^{(2)}$ and the excited-state hyperpolarizability $\alpha_{\xi \xi \xi, \mathrm{e}}^{(2)}$ have opposite phases for a two-state system. The approximation that $\mathrm{C} 314$ can be treated as a two-state system derives from the fact that the SHG frequency at $2 \omega$ is resonant with the strongest transition, $\mathrm{S}_{0} \rightarrow \mathrm{S}_{1}$, in C314. It therefore follows that the transitions to other states must be small and are, therefore, neglected.

\section{Experimental}

The pump-probe, time-resolved, second harmonic generation (TRSHG) setup is built around a regeneratively amplified Ti: sapphire femtosecond oscillator (Clark MXR) that produces 130 fs pulses with energies of $\sim 1 \mathrm{~mJ}$ at $842 \mathrm{~nm}$ and a repetition rate of $1 \mathrm{kHz}$. Frequency-doubling a small portion of the fundamental in a $\mathrm{BBO}$ crystal is used to obtain the pump excitation light of $421 \mathrm{~nm}$ with energies of $0.7 \mu \mathrm{J}$ per pulse. The remainder of the Ti:sapphire laser output pumps an optical parametric amplifier (Clark MXR), yielding a tunable probe beam output with energies of $13 \mu \mathrm{J}$ per pulse at $858 \mathrm{~nm}$. We note that the probe beam is two-photon-resonant with the coumarin 314 dye molecules at their respective interfaces. The polarization of the linearly polarized pump beam is controlled using a half-wave plate. The power of the $X$ and $Y$ linear polarized pump beam is carefully checked prior to experimentation to ensure that they are equal so as not to bias the in-plane distribution of excited-state molecules. The pump beam is incident normal to the sample surface. The probe beam is polarized at $45^{\circ}$ in the $X-Z$ plane and incident on the sample surface at $70^{\circ}$ from normal. The sample is contained in a shallow Teflon beaker mounted on a stage rotating at $2.5 \mathrm{rpm}$ to minimize heating and degradation effects. The $\chi_{x z x}^{(2)}$ element of the resultant second harmonic signal is selected by an S-oriented Glan-Taylor polarizer with a short wave pass glass filter and several apertures used to block the fundamental prior to the collection optics. The $X$-axis is in the plane of the interface and perpendicular to the plane of incidence, with the $Z$-axis defined as normal to the interface. The generated SH signal is focused into a $1 / 4 \mathrm{~m}$ monochromator (Jarell Ash) and detected by a PMT (Hamamatsu).

Laser grade coumarin 314 (Acros) was recrystallized from ethanol and sublimed in vacuo at $40{ }^{\circ} \mathrm{C}$. A $15 \mu \mathrm{M}$ saturated solution was made using ultrapure water (resistivity $=18.2$ $\mathrm{M} \Omega \cdot \mathrm{cm}$, Millipore Corp). GC-grade SDS (Fluka) was purified by dissolving in hot $95 \% \mathrm{EtOH}(14 \mathrm{~mL} / \mathrm{g})$ then filtering and cooling. This process was repeated five times, followed by drying overnight in a vacuum desiccator. The monolayers were prepared by dissolving SDS in $12.5 \mathrm{~mL}$ of the coumarin 314 solution, transferring the solution to a shallow Teflon beaker, and allowing the solution to stand for $30 \mathrm{~min}$ prior to the start of laser experimentation to allow for complete monolayer formation. The Teflon beakers were cleaned prior to use by immersion in freshly prepared piranha solution for $20 \mathrm{~min}$ and then rinsed with copious amounts of ultrapure water to remove any trace organics.

\section{Results and Discussion}

The results of the $X$ and $Y$ linearly polarized pumping are shown on the left side of Figure 4 for the two interfaces investigated. The normalized SHG electric field (square root of the SHG intensity) is plotted vs the pump delay. The initial constant signal level at negative delay times before the pump arrives is due to the contribution of the ground-state molecules to the SH intensity. Immediately upon pumping, the signal drops due to ground-state bleaching and cancelations due to the newly excited-state molecules having a phase for $\chi_{x z x}^{(2)}$ opposite that of the ground-state molecules. The drop in signal is greater for the $X$ linearly polarized pump than the $Y$ linearly polarized pumping because the $\chi_{x z x}^{(2)}$ element is more sensitive to the populations of ground- and excited-state molecules oriented along the $X$-axis than to the populations oriented along the $Y$-axis. The subsequent increase in the $\mathrm{SH}$ signal is due to the ultrafast solvation dynamics, which is followed by the slower rotational motions of the ground- and excited-state molecules moving toward their equilibrium orientational distributions. The $X$ - and $Y$-pump decay curves eventually reach the same signal 


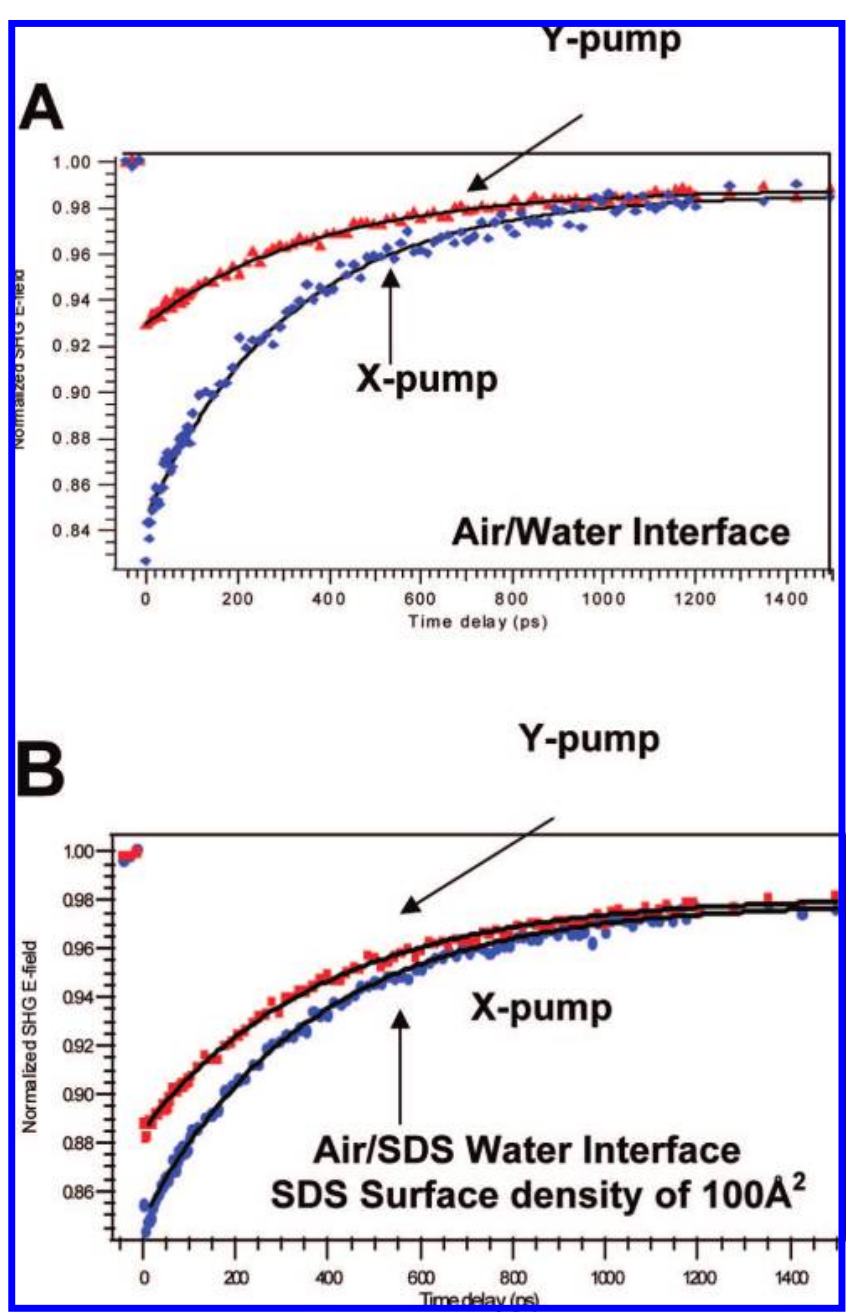

Figure 4. In-plane orientational motion of coumarin 314 at (A) the air/water interface, (B) the SDS $100 \AA^{2}$ per molecule/water interface. The single exponential fits to the $X$ - and $Y$-pump curves yield differing rotational kinetics since the linearly polarized $X$ - and $Y$-pumps do not contribute identically to $\chi_{x z x}^{(2)}$.

level when orientational equilibrium is reached and the in-plane isotropy is reestablished. The reason that the $\mathrm{SH}$ signal does not regain its prepump level on the time scale of the plots in Figure 4 is that the population of excited-state molecules have an excited-state lifetime of $4.5 \mathrm{~ns}^{7}{ }^{7}$ However, the same equilibrium orientational distribution is reached regardless of the initial photoselection due to pump polarization. This is confirmed by the observation that the $X$ and $Y$ curves recover to the same level at longer times (Figure 4); the circularly pumped dynamics recovers to the same level, as well (Figure 5). It is shown also that the average of the kinetic curves for the $X$ - and $Y$-pumping is equal to circularly polarized kinetic curves, as required by the addition of eqs 1 and 2, which yields eq 3 . For the air/ water interface, the average for the out-of-plane rotation time obtained by combining the $X$ and $Y$ kinetics is $333 \pm 13$ ps, in agreement with the out-of-plane orientational relaxation time obtained with circular pumping of $336 \pm 6$ ps. At a surface coverage of $100 \AA^{2}$ per SDS molecule, the average of the $X$ and $Y$ experimental curves is $365 \pm 9 \mathrm{ps,} \mathrm{which} \mathrm{is} \mathrm{in} \mathrm{agreement}$ with the relaxation time of $383 \pm 9$ ps obtained from circular pumping.

As shown in eq 13, the in-plane dynamics can be obtained by taking the ratio of the time-dependent second harmonic fields $E_{X}^{2 \omega}(t)$ and $E_{Y}^{2 \omega}(t)$. The result for the in-plane rotational motion of C314 at the neat air/water interface is $304 \pm 16 \mathrm{ps,} \mathrm{and} \mathrm{at} \mathrm{a}$ surface coverage of $100 \AA^{2}$ per molecule, the time constant is $348 \pm 12$ ps.

It is surprising that there is not a greater slowing of the C314 rotation dynamics in the presence of charged SDS surfactant, as compared with the neat air/water interface, for both in- and out-of-plane rotational motions. One might expect that an increased polar alignment of molecules induced at a charged surfactant interface would increase the frictional forces experienced by an interfacial solute molecule, thereby significantly slowing its rotational motions. A polar alignment of water molecules at charged interfaces has been observed at metal electrodes by X-ray scattering ${ }^{42}$ and surface-enhanced IR spectroscopy. ${ }^{43,44}$ Evidence for the changes in the hydrogen bonding structure near the charged interface were obtained using vibrational sum frequency generation spectroscopy of water at a charged quartz surface ${ }^{2,37}$ and at a charged surfactant monolayer air/water interface. ${ }^{3-5}$ The presence of the negatively charged surfactant head groups at the interface results in a large electrostatic field $\left(\sim 10^{8} \mathrm{~V} / \mathrm{m}\right)$, which partially aligns the water molecules. ${ }^{45}$ SFG studies of the OH-SS-S peak (3100-3300 $\mathrm{cm}^{-1}$ ) of interfacial water has suggested that water in the presence of the negatively charged SDS experiences higher $\mathrm{H}$-bond ordering, thereby yielding some water structures that are "icelike" at the interface. ${ }^{3}$ There are other studies of the neat air/water interface that claim that the interface is more structurally homogeneous than previously thought. ${ }^{46}$ In addition to the perturbation by SDS on the vibrational SFG spectra, there are other findings that established significant effects of a charged surfactant on equilibrium and dynamic phenomena. With respect to the latter, we have observed that that the presence of a negatively charged surfactant monolayer at the air/water interface markedly slows the solvation dynamics. ${ }^{6,33}$ The effects of SDS on equilibrium phenomena include the change in the C314 orientation from a polar angle $(\theta)$ of $67^{\circ}$ at the neat air/water interface to $45^{\circ}$ at the air/100 $\AA$ SDS interface, ${ }^{39}$ changes in the electronic spectra obtained from SHG measeurements ${ }^{6}$ (Figure 6), and changes in the air/SDS-water phase diagram ${ }^{6}$ (Figure 2). Considering that these are significant changes, which indicate a strong perturbation due to the SDS surfactant monolayer, it is surprising that there are such small changes in the in- and out-of-plane rotational relaxation times.

Although there is only a modest slowing of the in-plane rotation time for $\mathrm{C} 314$ at the air/water interface vs the SDS air/water interface, we note that for both interfaces, the in-plane air/water interfacial rotation time of $304 \pm 8$ ps is slower than the rotational relaxation of $\mathrm{C} 314$ molecule in bulk water (262 $\pm 10 \mathrm{ps}) .{ }^{40}$ We previously reported a similar slowing of the rotational dynamics of rhodamine $6 \mathrm{G}$ at the air/water interface. ${ }^{38}$ These results indicate that the interfacial friction for the adsorbates is considerably larger than in bulk aqueous solution.

Other studies have been carried out on molecular rotational dynamics at liquid interfaces that measured the decay in the fluorescence anisotropy of interfacial molecules. To minimize contributions from molecules in the bulk relative to the fluorescence from the interfacial population, the photoexcitation light was incident at angles that correspond to total internal reflection. In this way, the photoexcitation pulse penetrates only the bulk liquid, which contains fluorophores, to roughly 1000 $\AA$. It is only with molecules that can achieve a large interfacial population at very low bulk concentrations that it is possible to neglect fluorescence from the bulk molecules. Acridine orange at the aqueous/hexadecane interface satisfies this restriction. However, with few exceptions, the fluorescence from the bulk media cannot be separated from the interfacial adsorbate 


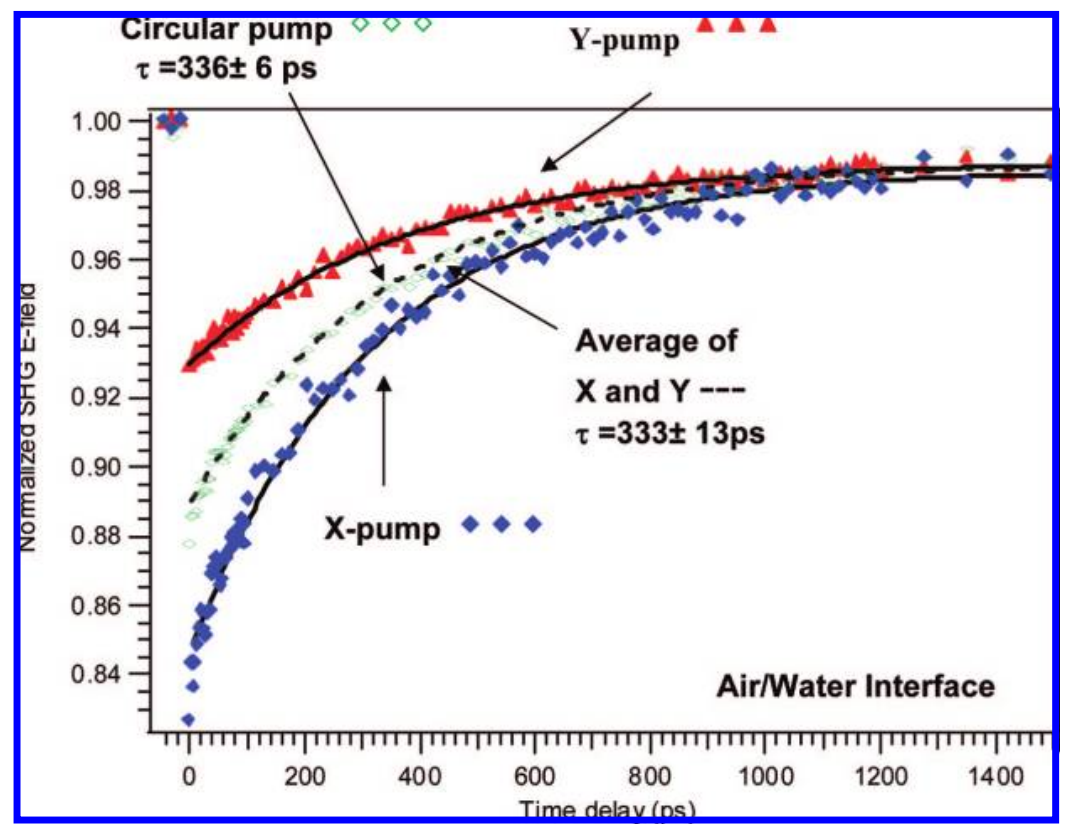

Figure 5. The average of the $X$ and $Y$ linear polarized pumping decay curves is equal to the circularly polarized kinetic curve. (A) The $X$ - and $Y$-polarized linear pump induced TRSHG decay data is shown. The solid lines are fits to a single exponential of the normalized second harmonic electric field vs the picosecond time delay. The average of the $X$ and $Y$ experimental data is shown as the dashed line; the single exponential fit to this curve yields a recovery time of $333 \pm 13$ ps. Also shown is circular polarized pumping of C314 at the air/water interface, represented by green triangles, which maps almost exactly onto the average of the $X$ and $Y$ data (dashed line).

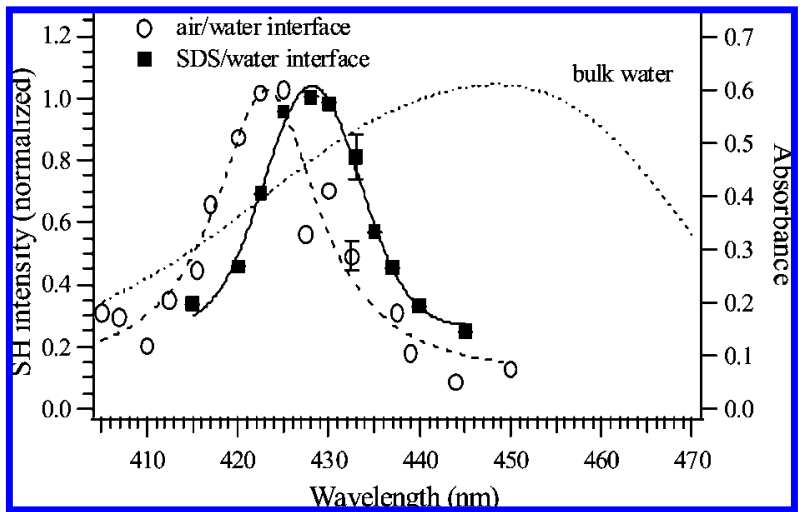

Figure 6. SHG spectra of C314 at the (ם) SDS air/water interface (surface coverage of $100 \AA^{2} / S D S$ molecule) and at the $(O)$ neat air/ water ${ }^{6}$ interface. The linear absorption spectrum of $\mathrm{C} 314$ in bulk water is also shown.

fluorescence. Using the fluorescence method, the out-of-plane motion of acridine orange, a cationic dye, at the aqueous/ hexadecane interface was found to be comparable., ${ }^{9,13}$ There have been other orientational dynamics studies of fluorescent chromophores attached to long hydrocarbon chains at the air/aqueous and a toluene/aqueous interface. ${ }^{10,11}$ Comparison with bulk orientational dynamics is not feasible because of the insolubility of the long-chain molecules.

There has been one SHG study of interfacial rotation dynamics that observed a faster interfacial rotation time than in the bulk liquid. ${ }^{8}$ The rotational relaxation time of the dianion eosin $\mathrm{B}$ at the air/aqueous interface was determined to be 90 ps, whereas in the bulk, it is 350 ps. This faster time was attributed to reduced interfacial hydrogen bonding with water at the interface, as compared with the bulk solution. We note that $\mathrm{C} 314$ and rhodamine $6 \mathrm{G}$ have significantly fewer sites for hydrogen bonding than the dianionic eosin B molecule, whose structure contains numerous carbon- and nitrogen-atom-bonded oxygens with available lone pair electrons suitable for hydrogen bonding with solvent water molecules.
On the theoretical side, molecular dynamics simulations of C314 at the air/water interface have yielded a number of equilibrium and dynamics properties. ${ }^{48,49}$ The calculated orientation of $\mathrm{C} 314$ is $\sim 80^{\circ}$, in good agreement with experiment, $\sim 67-80^{\circ} .6,7$ Calculations of the ground- and excited-state dipole moments were also found to be in reasonable agreement with the measured values. However, simulations of dynamical processes for $\mathrm{C} 314$ at the air/aqueous interface are more difficult to carry out. The out-of-plane rotational temporal correlation relaxation times obtained from simulations were roughly an order of magnitude faster than the experimental results reported in the laboratory. The correlation time expression used in the molecular dynamics simulation involved the function $\delta[\cos \theta(\mathrm{t})]$ $\delta[\cos \theta(0)]$, whereas the SHG correlation time expression will have a different dependence on $\theta$. Simulations of the in-plane rotational of $\mathrm{C} 314$ were found to be rather complicated, more so than for out-of-plane motions. ${ }^{47}$ The physical picture of the C314 in-plane rotation described a rapid large-scale motion in which $\mathrm{C} 314$ projected almost perpendicular to the interface and returned to an almost flat orientation in a time scale of several hundred picoseconds. This time scale is roughly what was obtained from the SHG measurements described here. However, their preliminary results showed that the in-plane rotation was slower than the out-of-plane rotational relaxation, which is contrary to our SHG findings. There have also been simulations that investigate the effects of the charged surfactant SDS on the rotation of $\mathrm{C} 314$ at the SDS air/aqueous interface. ${ }^{49}$ The simulation results showed a slowing of the C314 rotational relaxation when SDS was at the interface, which is in agreement with the results obtained from the SHG experiments reported here. The magnitude of the effect of SDS surface density on the rotation time was found to be greater than that obtained from SHG experiments.

In other simulations, the orientational relaxation of the probe molecule, $N-N^{\prime}$-diethyl- $p$-nitroaniline at the air/water interface yielded rotational relaxation times that are faster than in bulk water. ${ }^{50}$ This faster interfacial rotational relaxation time was also 
found in studies at the liquid/liquid interface, modeled by two identical 6-12 Lennard-Jones liquids with solvent molecule masses identical to $\mathrm{CCl}_{4}$ and using solute molecules consisting of two joined solvent molecules. The results described a faster solute rotation at the interface, which slows to approach bulk values as the surface tension is decreased with surfactant addition. ${ }^{51}$ In both cases, the faster rotation time was attributed to the lower density of solvent molecules at interfaces. The disagreement with experimental results for $\mathrm{C} 314$, rhodamine $6 \mathrm{G}$, and acridine orange, but not for eosin B (in which hydrogen bonding was a key factor), could be due to the fact that the molecules used in the simulations are considerably different in structure from the organic dyes used in the SHG and fluorescence experiments.

\section{Conclusion}

The in-plane rotational dynamics of the molecular probe coumarin 314 (C314) at the negatively charged surfactant sodium dodecyl sulfate (SDS)/aqueous interface was measured using femtosecond time-resolved, second-harmonic spectroscopy. At an SDS density of $100 \AA^{2}$ per SDS molecule, the surfactant is in the liquid condensed phase region of the surface pressure phase diagram, and thus, the monolayer coverage experienced by C314 is homogeneous. The in-plane orientational time constant for the $100 \AA^{2}$ SDS interface was found to be slower than at the neat air/water interface. Comparing the inplane and out-of-plane motions, we found that the out-of-plane rotation was slower, but only slightly $(10-15 \%)$ than the inplane rotation for both the air/100 $\AA^{2}$ SDS-water interface and the neat air/water interfaces. In all cases, the interface rotation times are significantly slower than the relaxation in bulk water. Although the C314-equilibrium interfacial orientation angle, surface spectra, SDS surfactant/C314 aqueous solution phase diagram, and solvation dynamics are significantly affected by the SDS surfactant, surprisingly, neither the in-plane nor the out-of-plane rotations are greatly affected. This rotational insensitivity to the anionic SDS surfactant has implications with respect to molecular rotational motions at aqueous/biological cell membrane interfaces, most of which are known to contain negatively charged phospholipids.

Acknowledgment. The authors thank the National Science Foundation, the Chemical Sciences, Geosciences and Biosciences Division; and Office of Basic Energy Sciences, Office of Science, U.S. Department of Energy; and DTRA (W911NF07-1-0116).

\section{References and Notes}

(1) Du, Q.; Freysz, E.; Shen, Y. R. Science 1994, 264, 826.

(2) Gopalakrishnan, S.; Liu, D.; Allen, H. C.; Kuo, M.; Schultz, M. J. Chem. Rev. 2006, 106, 1155.

(3) Eisenthal, K. B. Chem. Rev. 2006, 106, 1462.

(4) Gragson, D. E.; McCarty, B. M.; Richmond, G. L. J. Am. Chem. Soc. 1997, 119, 6144. 3847.

(5) Gragson, D. E.; Richmond, G. L. J. Phvs. Chem. B 1998, 102,

(6) Benderskii, A. V.; Eisenthal, K. B. J. Phys. Chem. A 2002, 106, 7482
(7) Zimdars, D.; Dadap, J. I.; Eisenthal, K. B.; Heinz, T. F. J. Phvs. Chem. B 1999, 103, 3425 .

(8) Antoine, R.; Tamburello-Luca, A. A.; Hebert, P.; Brevet, P. F.; Girault, H. H. Chem. Phvs. Lett. 1998, 288, 138.

(9) Piasecki, D. A.; Wirth, M. J. J. Am. Chem. Soc. 1993, 97, 7700.

(10) Miura, A.; Tamai, N. Chem. Phvs. Lett. 2000, 328, 23.

(11) Tsukahara, S.; Yamada, Y.; Watarai, H. Langmuir 2000, 16, 6787.

(12) Burbage, J. D.; Wirth, M. J. J. Phys. Chem. 1992, 96, 5943.

(13) Wirth, M. J.; Burbage, J. D. J. Phvs. Chem. 1992, 96, 9022.

(14) Eisenthal, K. B. Chem. Rev. 1996, 96, 1343.

(15) Corn, R. M.; Higgins, D. A. Chem. Rev. 1994, 94, 107.

(16) Richmond, G. L.; Robinson, J. M.; Shannon, V. L. Prog. Surf. Sci. 1988, $28,1$.

(17) Battacharyya, K.; Sitzmann, E. V.; Eisenthal, K. B. J. Chem. Phys. 1988, $89,3376$.

(18) Zimdars, D.; Dadap, J. I.; Eisenthal, K. B.; Heinz, T. F. Chem. Phvs. Lett. 1999, 301, 112.

(19) Rinuy, J.; Brevet, P. F.; Girault, H. H. Biophys. J. 1999, 77, 3350.

(20) Dounce, S. M.; Yang, M.; Dai, H. L. Phys. Rev. B 2003, 67.

(21) Dounce, S. M.; Yang, M.; Dai, H. L. Surf. Sci. 2004, 565, 27.

(22) Shang, X. M.; Benderskii, A. V.; Eisenthal, K. B. J.Phvs. Chem. B 2001, 105, 11578 .

(23) Polizzi, M. A.; Plocinik, R. M.; Simpson, G. J. J. Am. Chem. Soc. 2004, 126, 5001.

(24) Lantz, J. M.; Corn, R. M. J. Phvs. Chem. 1994, 98, 9387.

(25) Jen, S. H.; Dai, H. L. J. Phvs. Chem. B 2006, 110, 23000.

(26) Mifflin, A. L.; Gerth, K. A.; Geiger, F. M. J. Phvs. Chem. A 2003, 107,9620 .

(27) Steel, W. H.; Damkaci, F.; Nolan, R.; Walker, R. A. J. Am. Chem. Soc. 2002, 124, 4824.

(28) Kott, K.; Higgins, D.; McMahon, R.; Corn, R. M. J. Am. Chem. Soc. 1993, 115,5342 .

(29) Conboy, J. C.; Richmond, G. L. Electrochim. Acta 1995, 40, 2881. (30) Pant, D.; Le Guennec, M.; Illien, B.; Girault, H. H. Phvs. Chem. Chem. Phvs. 2004, 6, 3140.

(31) Nagatani, H.; Piron, A.; Brevat, P. F.; Fermin, D. J.; Girault, H. H. Langmuir 2002, 18, 6647.

(32) Stryer, L. Biochemistry, 4th ed.; W. H. Freeman: New York, 1995. (33) Benderskii, A. V.; Eisenthal, K. B. J. Phvs. Chem. B 2001, 105, 6698.

(34) Miranda, P. B.; Shen, Y. R. J. Phvs. Chem. B 1999, 103, 3292.

(35) Bell, G. R.; Bain, C. D.; Ward, R. N. J. Chem. Soc., Faradav Trans. 1996, 92, 515.

(36) Chattoraj, D. K.; Birdi, K. S. Adsorption and the Gibbs Surface Excess; Plenum: New York, 1984.

(37) Du, Q.; Superfine, R.; Freysz, E.; Shen, Y. R. Phvs. Rev. Lett. 1993, $70,2313$.

(38) Castro, A.; Sitzmann, E. V.; Zhang, D.; Eisenthal, K. B. J. Phys. Chem. 1991, 95, 6752.

(39) Nguyen, K. T.; Shang, X.; Eisenthal, K. B. J. Phys. Chem. B 2005, 109, submitted.

(40) McArthur, E. A. Ph. D. thesis.

(41) Goh, M. C.; Hicks, J. M.; Kemnitz, K.; Pinto, G. R.; Bhattacharyya,

K.; Heinz, T. F.; Eisenthal, K. B. J. Phvs. Chem. 1988, 92, 5074.

(42) Toney, M. F.; Howard, J. N.; Richer, J.; Borges, G. L.; Gordon, J. G.; Melroy, O. R.; Wiesler, D. G.; Yee, D.; Sorensen, L. B. Nature 1994, $368,444$.

(43) Habib, M. A.; Bockris, J. O. Langmuir 1986, 2, 388.

(44) Ataka, K.; Yotsuyanagi, T.; Osawa, M. J. Phvs. Chem. 1996, 100, 10664 .

(45) Zhao, X. L.; Ong, S. W.; Eisenthal, K. B. Chem. Phys. Lett. 1993, 202, 513.

(46) Sovago, M.; Campen, R. K.; Wurpel, G. W. H.; Muller, M.; Bakker, H. J.; Bonn, M. Phvs. Rev. Lett. 2008, 100, 173901.

(47) Chou, S. H.; Wirth, M. J. J. Phys. Chem. 1989, 93, 7694.

(48) Pantano, D. A.; Laria, D. J. Phys. Chem. B 2003, 107, 2971.

(49) Pantano, D. A.; Sonoda, M. T.; Skaf, M. S.; Laria, D. J. Phvs. Chem. B 2005, 109, 7365 .

(50) Michael, D.; Benjamin, I. J. Phvs. Chem. B 1998, 102, 5145.

(51) Hill, A. W.; Benjamin, I. J. Phys. Chem. B 2004, 108, 15443.

JP807273V 